\title{
Estimation of the Parameters of a Bivariate Geometric Distribution
}

\author{
U.J. Dixit and S. Annapurna \\ Department of Statistics, University of Mumbai, Mumbai-India \\ ulhasdixit@yahoo.co.in; annapurnas2170@gmail.com,annapurna.s@xaviers.edu
}

Received 22 August 2014

Accepted 14 May 2015

\begin{abstract}
The uniformly minimum variance unbiased estimators (UMVUE) of the parameters and reliability functions of a bivariate geometric distribution(BGD) have been derived.The exact variances of the maximum likelihood estimator (MLE) and of UMVUE have been derived and the corresponding mean square errors have been compared.It is found that in some cases UMVUE is better and in some cases MLE is better with respect to the mean square errors. In the final section an example of actual data from the game Cricket's Indian Premium League 2014 (IPL 2014) has been given.

Keywords: Bivariate Geometric Distribution, Maximum Likelihood Estimators,Uniformly Minimum Variance Unbiased Estimators, Reliability Functions, cricket.
\end{abstract}

\section{Introduction}

The discrete bivariate geometric distribution (BGD) has applications in survival analysis and reliability theory. Any system with two or more individual components like twin engines of an airplane or paired organs in a human body can be studied by using such reliability models. The twocomponent system can be visualized as comprising of three independent components, the third being the interdependence of the two components.Phatak and Sreehari (1981) introduced a version of BGD as a stochastic model for giving the distribution of good and marginally good items that are produced before a bad item is produced by a production unit.

Downtown(1970) also described the BGD which arises in a shock model with two components.Suppose that the number of shocks suffered by each component before failure can be represented by a population in which $p_{1}$ and $p_{2}$ are proportions of shocks affecting the first and second components respectively without failure and a proportion $p_{3}=\left(1-p_{1}-p_{2}\right)$ of the shocks leading to failure of both components,see Downtown(1970).

Let $\mathrm{X}$ be number of shocks to component 1 prior to the first failure.Let $\mathrm{Y}$ be number of shocks to component 2 prior to the first failure. 
The joint probability mass function of $(\mathrm{X}, \mathrm{Y})$ is given by Kocherlakote (1992) as

$$
P(X=x, Y=y)=\left\{\begin{array}{cc}
\left(\begin{array}{c}
x+y \\
x
\end{array}\right) p_{1}^{x} p_{2}^{y} p_{3} ; x=0,1,2, \cdots, y=0,1,2, \cdots \\
0<p_{1}<1 ; 0<p_{2}<1 ; p_{3}=1-p_{1}-p_{2} \\
0 \quad \text { otherwise }
\end{array}\right.
$$

Krishna and Pundir(2009) estimated the parameters of BGD as given in (1.1).Krishna and Pundir(2009) have derived MLE of the parameters and the reliability functions. Further they have derived asymptotic expected values of MLE and reliability functions.But they have not obtained the UMVUE of the parameters of the BGD. In this paper we obtain the UMVUE of the parameters and the reliability functions.Further we derive the exact expression of its variance.Morover we have compared the mean square errors of MLE and UMVUE. It is found that in some cases UMVUE is better and in some cases MLE is better with respect to its mean square errors.

\section{Pre-Requisite Results}

A. Suppose in an urn there are $\mathrm{W}+\mathrm{R}+\mathrm{G}+1$ balls such that $\mathrm{W}$ are white, $\mathrm{R}$ are red, $\mathrm{G}$ are orange and one of a different colour.We add $\mathrm{x}$ balls of white colour to the urn and remove $\mathrm{x}$ balls of red or orange colour.If we select a count of $\mathrm{R}$ balls from the urn such that $\mathrm{x}$ are white and the remaining are red or orange.

$$
\sum_{x=0}^{R}\left(\begin{array}{c}
W+x \\
x
\end{array}\right)\left(\begin{array}{c}
R+G-x \\
R-x
\end{array}\right)=\left(\begin{array}{c}
W+R+G+1 \\
R
\end{array}\right) \quad G>0
$$

(2.1) can be easily proved by elementary algebraic methods..

B. Hypergeometric Functions.

$$
\begin{gathered}
\text { 1. }{ }_{m} F_{n}\left(a_{1}, a_{2}, \ldots, a_{m} ; b_{1}, b_{2}, \ldots, b_{n} ; p_{2}\right) \\
=\sum_{j=0}^{\infty} \frac{\Gamma\left(a_{1}+j\right)}{\Gamma a_{1}} \frac{\Gamma\left(a_{2}+j\right)}{\Gamma a_{2}} \ldots \frac{\Gamma\left(a_{m}+j\right)}{\Gamma a_{m}} \frac{\Gamma b_{1}}{\Gamma\left(b_{1}+j\right)} \ldots \frac{\Gamma b_{n}}{\Gamma\left(b_{n}+j\right)} \frac{p_{2}^{j}}{j !} \\
\text { 2. }{ }_{m+2} F_{n+1}\left((a)^{m}, b, c ;(d)^{n}, e ; p_{2}\right) \\
=\sum_{j=0}^{\infty} \frac{(\Gamma(a+j))^{m}}{(\Gamma a)^{m}} \frac{\Gamma(b+j)}{\Gamma b} \frac{\Gamma(c+j)}{\Gamma c} \frac{(\Gamma d)^{n}}{(\Gamma(d+j))^{n}} \frac{\Gamma e}{\Gamma(e+j)} \frac{p_{2}^{j}}{j !} \\
=\sum_{m=0}^{\infty} \sum_{n=0}^{\infty} \frac{\Gamma(a+m+n)}{\Gamma(a)} \frac{\Gamma(b+m)}{\Gamma(b)} \frac{\Gamma(c+n)}{\Gamma(c)} \frac{\Gamma(d)}{\Gamma(d+m+n)} \frac{\Gamma(e)}{\Gamma(e+m)} \frac{p_{1}^{m}}{m !} \frac{p_{2}^{n}}{n !} \\
\text { Published by Atlantis Press } \\
\text { Copyright: the authors } \\
325
\end{gathered}
$$




\section{Main Results}

Theorem 3.1

The UMVUE of $p_{1}^{r_{1}} p_{2}^{r_{2}} p_{3}^{r_{3}}$

$$
=\frac{\left(\begin{array}{c}
s_{1}+n-r_{1}-r_{3}-1 \\
s_{1}-r_{1}
\end{array}\right)\left(\begin{array}{c}
s_{1}+s_{2}+n-r_{1}-r_{2}-r_{3}-1 \\
s_{2}-r_{2}
\end{array}\right)}{\left(\begin{array}{c}
s_{1}+n-1 \\
s_{1}
\end{array}\right)\left(\begin{array}{c}
s_{1}+s_{2}+n-1 \\
s_{2}
\end{array}\right)}
$$

where $S_{1}=\sum_{i=1}^{n} X_{i}$ and $S_{2}=\sum_{i=1}^{n} Y_{i}$

Proof: The joint distribution of $\left(X_{1} Y_{1}, X_{2} Y_{2}, \ldots, X_{n} Y_{n}\right)$ belongs to the exponential family and ( $S_{1}$, $S_{2}$ ) is sufficient and complete for (1.1). Hence by using Lehmann Scheffe's theorem we can get UMVUE of $p_{1}^{r_{1}} p_{2}^{r_{2}} p_{3}^{r_{3}}$.

The joint distribution of $\left(S_{1}, S_{2}\right)$ is given by

$$
P\left(S_{1}=s_{1}, S_{2}=s_{2}\right)=\left\{\begin{array}{cc}
\left(\begin{array}{c}
s_{1}+n-1 \\
s_{1}
\end{array}\right)\left(\begin{array}{c}
s_{1}+s_{2}+n-1 \\
s_{2}
\end{array}\right) p_{1}{ }^{s_{1}} p_{2}{ }^{s_{2}} p_{3}{ }^{n} ; s_{1}=0,1,2, \ldots, \\
\\
s_{2}=0,1,2 \ldots, \\
0<p_{1}<1 ; 0<p_{2}<1 \\
p_{3}=1-p_{1}-p_{2} \\
; \text { otherwise }
\end{array}\right.
$$

Let $\phi\left(s_{1}, s_{2}\right)$ be the UMVUE of $p_{1}^{r_{1}} p_{2}^{r_{2}} p_{3}^{r_{3}}$

$$
\begin{gathered}
E\left(\phi\left(s_{1}, s_{2}\right)=\sum_{s_{1}=0}^{\infty} \sum_{s_{2}=0}^{\infty} \phi\left(s_{1}, s_{2}\right)\left(\begin{array}{c}
s_{1}+n-1 \\
s_{1}
\end{array}\right)\left(\begin{array}{c}
s_{1}+s_{2}+n-1 \\
s_{2}
\end{array}\right) p_{1}{ }^{s_{1}} p_{2}{ }^{s_{2}} p_{3}{ }^{n}\right. \\
=p_{1}{ }^{r_{1}} p_{2}{ }^{r_{2}} p_{3}{ }^{r_{3}} \\
\sum_{s_{1}=r_{1}}^{\infty} \sum_{s_{2}=r_{2}}^{\infty} \phi\left(s_{1}, s_{2}\right)\left(\begin{array}{c}
s_{1}+n-1 \\
s_{1}
\end{array}\right)\left(\begin{array}{c}
s_{1}+s_{2}+n-1 \\
s_{2}
\end{array}\right) p_{1}{ }^{s_{1}-r_{1}} p_{2}{ }^{s_{2}-r_{2}} p_{3}{ }^{n-r_{3}}=1
\end{gathered}
$$

Therefore

$$
\phi\left(s_{1}, s_{2}\right)=\frac{\left(\begin{array}{c}
s_{1}+n-r_{1}-r_{3}-1 \\
s_{1}-r_{1}
\end{array}\right)\left(\begin{array}{c}
s_{1}+s_{2}+n-r_{1}-r_{2}-r_{3}-1 \\
s_{2}-r_{2}
\end{array}\right)}{\left(\begin{array}{c}
s_{1}+n-1 \\
s_{1}
\end{array}\right)\left(\begin{array}{c}
s_{1}+s_{2}+n-1 \\
s_{2}
\end{array}\right)}
$$




\section{$\underline{\text { Particular Cases }}$}

1. $r_{2}=r_{3}=0$

$$
\widehat{p_{1}^{r_{1}}}=\frac{\left(\begin{array}{c}
s_{1}+n-r_{1}-1 \\
s_{1}-r_{1}
\end{array}\right)\left(\begin{array}{c}
s_{1}+s_{2}+n-r_{1}-1 \\
s_{2}
\end{array}\right)}{\left(\begin{array}{c}
s_{1}+n-1 \\
s_{1}
\end{array}\right)\left(\begin{array}{c}
s_{1}+s_{2}+n-1 \\
s_{2}
\end{array}\right)}
$$

2. $r_{1}=r_{3}=0$

$$
\widehat{p_{2}{ }^{r_{2}}}=\frac{\left(\begin{array}{c}
s_{1}+n-1 \\
s_{1}
\end{array}\right)\left(\begin{array}{c}
s_{1}+s_{2}+n-r_{2}-1 \\
s_{2}-r_{2}
\end{array}\right)}{\left(\begin{array}{c}
s_{1}+n-1 \\
s_{1}
\end{array}\right)\left(\begin{array}{c}
s_{1}+s_{2}+n-1 \\
s_{2}
\end{array}\right)}
$$

3. $r_{1}=r_{2}=0$

$$
\widehat{p_{3} r_{3}}=\frac{\left(\begin{array}{c}
s_{1}+n-r_{3}-1 \\
s_{1}
\end{array}\right)\left(\begin{array}{c}
s_{1}+s_{2}+n-r_{3}-1 \\
s_{2}
\end{array}\right)}{\left(\begin{array}{c}
s_{1}+n-1 \\
s_{1}
\end{array}\right)\left(\begin{array}{c}
s_{1}+s_{2}+n-1 \\
s_{2}
\end{array}\right)}
$$

Similarly we can obtain UMVUE for $p_{1}^{r_{1}} p_{2}^{r_{2}} p_{3}^{r_{3}}$ for different values of $r_{1}, r_{2}$ and $r_{3}$.

Theorem 3.2

$$
\begin{aligned}
V\left(\phi\left(s_{1}, s_{2}\right)\right) & =\frac{\Gamma n \Gamma\left(r_{1}+1\right) \Gamma\left(r_{2}+1\right)}{\Gamma\left(n+r_{1}+r_{2}\right)} p_{1}^{r_{1}} p_{2}^{r_{2}} p_{3}^{n} \\
& \times F\left(\left(\left(n-r_{3}\right)_{m+n}\right)^{2},\left(r_{1}+1\right)_{m},\left(r_{2}+1\right)_{n} ;\left(r_{1}+r_{2}+n\right)_{m+n},(1)_{m},(1)_{n} ; p_{1}, p_{2}\right) \\
& -p_{1}^{2 r_{1}} p_{2}^{2 r_{2}} p_{3}^{2 r_{3}}
\end{aligned}
$$

Proof:

$$
\begin{aligned}
& E\left(\phi\left(s_{1}, s_{2}\right)^{2}=\sum_{s_{1}=r_{1}}^{\infty} \sum_{s_{2}=r_{2}}^{\infty}\left(\phi\left(s_{1}, s_{2}\right)\right)^{2}\left(\begin{array}{c}
s_{1}+n-1 \\
s_{1}
\end{array}\right)\left(\begin{array}{c}
s_{1}+s_{2}+n-1 \\
s_{2}
\end{array}\right) p_{1}{ }^{s_{1}} p_{2}{ }^{s_{2}} p_{3}{ }^{n}\right. \\
& =\sum_{s_{1}=r_{1}}^{\infty} \frac{\left[\left(\begin{array}{c}
s_{1}+n-r_{1}-r_{3}-1 \\
s_{1}-r_{1}
\end{array}\right)\right]^{2}}{\left(\begin{array}{c}
s_{1}+n-1 \\
s_{1}
\end{array}\right)} p_{1}^{s_{1}} p_{3}^{n} \sum_{s_{2}=r_{2}}^{\infty} \frac{\left[\left(\begin{array}{c}
s_{1}+s_{2}+n-r_{1}-r_{3}-1 \\
s_{2}-r_{2}
\end{array}\right)\right]^{2}}{\left(\begin{array}{c}
s_{1}+s_{2}+n-1 \\
s_{2}
\end{array}\right)} p_{2}^{s_{2}}
\end{aligned}
$$




$$
=\sum_{s_{1}=r_{1}}^{\infty} \frac{\left[\left(\begin{array}{c}
s_{1}+n-r_{1}-r_{3}-1 \\
s_{1}-r_{1}
\end{array}\right)\right]^{2}}{\left(\begin{array}{c}
s_{1}+n-1 \\
s_{1}
\end{array}\right)} p_{1}^{s_{1}} p_{3}^{n} \sum_{j=0}^{\infty} \frac{\left[\left(\begin{array}{c}
s_{1}+j+n-r_{1}-r_{3}-1 \\
j
\end{array}\right)\right]^{2}}{\left(\begin{array}{c}
s_{1}+j+r_{2}+n-1 \\
j+r_{2}
\end{array}\right)} p_{2}^{j+r_{2}}
$$

Consider

$$
\begin{aligned}
\sum_{j=0}^{\infty} \frac{\left.\left(\begin{array}{c}
s_{1}+j+n-r_{1}-r_{3}-1 \\
j
\end{array}\right)\right)^{2}}{\left(\begin{array}{c}
s_{1}+j+r_{2}+n-1 \\
j+r_{2}
\end{array}\right)} p_{2}^{j+r_{2}} & =\left(p_{2}\right)^{r_{2}} \frac{\left(s_{1}+n-1\right) ! r_{2} !}{\left(s_{1}+r_{2}+n-1\right) !} \\
& \times{ }_{3} F_{2}\left(\left(s_{1}+n-r_{1}-r_{3}\right)^{2}, r_{2}+1 ; s_{1}+r_{2}+n, 1 ; p_{2}\right)
\end{aligned}
$$

Hence

$$
\begin{gathered}
E\left(\phi\left(s_{1}, s_{2}\right)\right)^{2}=\sum_{k=0}^{\infty} \frac{\Gamma\left(n-r_{3}+k\right) \Gamma\left(n-r_{3}+k\right) \Gamma\left(r_{1}+1+k\right) \Gamma n \Gamma\left(r_{2}+1\right)}{\Gamma\left(n-r_{3}\right) \Gamma\left(n-r_{3}\right) \Gamma(k+1) \Gamma(k+1) \Gamma\left(r_{1}+r_{2}+n+k\right)} p_{1}^{k+r_{1}} p_{2}^{r_{2}} p_{3}^{n} \\
\times{ }_{3} F_{2}\left(\left(k+n-r_{3}\right)^{2}, r_{2}+1 ; k+r_{1}+r_{2}+n, 1 ; p_{2}\right) \\
=\sum_{k=0}^{\infty} \sum_{j=0}^{\infty} \frac{\left(\Gamma\left(n-r_{3}+k+j\right)\right)^{2} \Gamma\left(r_{1}+1+k\right) \Gamma\left(r_{2}+1+j\right) \Gamma\left(r_{1}+r_{2}+n\right)}{\left(\Gamma\left(n-r_{3}\right)\right)^{2} \Gamma\left(r_{1}+1\right) \Gamma\left(r_{2}+1\right) \Gamma\left(r_{1}+r_{2}+n+k+j\right) \Gamma(k+1) \Gamma(j+1)} \frac{p_{1}^{k}}{k !} \frac{p_{2}^{j}}{j !} \\
=\frac{\Gamma n \Gamma\left(r_{1}+1\right) \Gamma\left(r_{2}+1\right)}{\Gamma\left(n+r_{1}+r_{2}\right)} p_{1}^{r_{1}} p_{2}^{r_{2}} p_{3}^{n} \\
\times F\left(\left(\left(n-r_{3}\right)_{(k+j)}\right)^{2},\left(r_{1}+1\right)_{k},\left(r_{2}+1\right)_{j} ;\left(r_{1}+r_{2}+n\right)_{k+j},(1)_{k},(1)_{j} ; p_{1}, p_{2}\right)
\end{gathered}
$$

Hence proved.

Krishna and Pundir(2009) obtained MLE of $p_{1}, p_{2}$ and $p_{3}$ as

$$
\widehat{p_{1}}=\frac{s_{1}}{n+s_{1}+s_{2}}, \widehat{p_{2}}=\frac{s_{2}}{n+s_{1}+s_{2}}, \widehat{p_{3}}=\frac{n}{n+s_{1}+s_{2}}
$$

By invariance property, MLE of $p_{1}^{r_{1}} p_{2}^{r_{2}} p_{3}^{r_{3}}$ is

$$
\widehat{p_{1}^{r_{1}} p_{2}^{r_{2}} p_{3}^{r_{3}}}=\frac{s_{1}^{r_{1}} s_{2}^{r_{2}} s_{3}^{r_{3}}}{\left(n+s_{1}+s_{2}\right)^{r_{1}+r_{2}+r_{3}}}
$$


Theorem 3.3 Variance of MLE of $p_{1}{ }^{r_{1}} p_{2}{ }^{r_{2}} p_{3}{ }^{r_{3}}=$

$$
\begin{aligned}
& \sum_{s_{1}=0}^{\infty} \sum_{s_{2}=0}^{\infty}\left(\frac{s_{1}^{r_{1}} s_{2}^{r_{2}} n^{r_{3}}}{\left(n+s_{1}+s_{2}\right)^{r_{1}+r_{2}+r_{3}}}\right)^{2}\left(\begin{array}{c}
s_{1}+n-1 \\
s_{1}
\end{array}\right)\left(\begin{array}{c}
s_{1}+s_{2}+n-1 \\
s_{2}
\end{array}\right) p_{1}^{r_{1}} p_{2}^{r_{2}} p_{3}^{n} \\
- & \left(\sum_{s_{1}=0}^{\infty} \sum_{s_{2}=0}^{\infty} \frac{s_{1}^{r_{1}} s_{2}^{r_{2}} n^{r_{3}}}{\left(n+s_{1}+s_{2}\right)^{r_{1}+r_{2}+r_{3}}}\left(\begin{array}{c}
s_{1}+n-1 \\
s_{1}
\end{array}\right)\left(\begin{array}{c}
s_{1}+s_{2}+n-1 \\
s_{2}
\end{array}\right) p_{1}^{r_{1}} p_{2}^{r_{2}} p_{3}^{n}\right)^{2}
\end{aligned}
$$

Thus Mean Square Error(MSE) of $\widehat{p_{1}^{r_{1}}} \widehat{p_{2}^{r_{2}}} \widehat{p_{3}^{r_{3}}}=$

$$
V\left(\widehat{p_{1}^{r_{1}}} \widehat{p_{2}^{r_{2}}} \widehat{p_{3}^{r_{3}}}\right)+\operatorname{Bias}\left(\widehat{p_{1}^{r_{1}}} \widehat{p_{2}^{r_{2}}} \widehat{p_{3}^{r_{3}}}\right)^{2}
$$

\section{Estimation of Reliability Functions}

The reliability function is given by $R(x, y)=P(X \geq x, Y \geq y)$

$$
\begin{gathered}
R(x, y)=1 ; x=0, y=0 \\
=\left(\frac{p_{1}}{1-p_{2}}\right)^{x} ; x=1,2,3, \ldots ; y=0 \\
=\left(\frac{p_{2}}{1-p_{1}}\right)^{y} ; x=0 ; y=1,2,3, \ldots
\end{gathered}
$$

$$
=\left(\frac{p_{1}}{1-p_{2}}\right)^{x}+\left(\frac{p_{2}}{1-p_{1}}\right)^{y}+\sum_{r=0}^{x-1} \sum_{s=0}^{y-1}\left(\begin{array}{c}
r+s \\
r
\end{array}\right) p_{1}^{r} p_{2}^{s} p_{3}-1 ; x=1,2,3, \ldots ; y=1,2,3, \ldots
$$

Consider

$$
\left(\frac{p_{1}}{1-p_{2}}\right)^{x}=p_{1}^{x} \sum_{j=0}^{\infty}\left(\begin{array}{c}
j+x-1 \\
x-1
\end{array}\right) p_{2}^{j}
$$

By putting $r_{1}=x ; r_{2}=j$ and $r_{3}=0$ in (3.1) the UMVUE of (4.1) is

$$
=\sum_{j=0}^{s_{2}} \frac{\left(\begin{array}{c}
j+x-1 \\
x-1
\end{array}\right)\left(\begin{array}{c}
s_{1}+n-x-1 \\
s_{1}-x
\end{array}\right)\left(\begin{array}{c}
s_{1}+s_{2}+n-x-j-1 \\
s_{2}-j
\end{array}\right)}{\left(\begin{array}{c}
s_{1}+n-1 \\
s_{1}
\end{array}\right)\left(\begin{array}{c}
s_{1}+s_{2}+n-1 \\
s_{2}
\end{array}\right)}
$$


By using (2.1), (4.4) becomes $=\frac{\left(\begin{array}{c}s_{1} \\ x\end{array}\right)}{\left(\begin{array}{c}s_{1}+n-1 \\ x\end{array}\right)}$

Similarly UMVUE of (4.2) becomes $=\frac{\left(\begin{array}{c}s_{2} \\ y\end{array}\right)}{\left(\begin{array}{c}s_{2}+n-1 \\ y\end{array}\right)}$

Using (3.1), UMVUE of (4.3) is $=\frac{\left(\begin{array}{c}s_{1} \\ x\end{array}\right)}{\left(\begin{array}{c}s_{1}+n-1 \\ x\end{array}\right)}+\frac{\left(\begin{array}{c}s_{2} \\ y\end{array}\right)}{\left(\begin{array}{c}s_{2}+n-1 \\ y\end{array}\right)}$
$+\sum_{r=0}^{x-1} \sum_{s=0}^{y-1} \frac{\left(\begin{array}{c}r+s \\ r\end{array}\right)\left(\begin{array}{c}s_{1}+n-r-2 \\ s_{1}-r\end{array}\right)\left(\begin{array}{c}s_{1}+s_{2}+n-r-s-2 \\ s_{2}-s\end{array}\right)}{\left(\begin{array}{c}s_{1}+n-1 \\ s_{1}\end{array}\right)\left(\begin{array}{c}s_{1}+s_{2}+n-1 \\ s_{2}\end{array}\right)}-1$

Using Theorem (3.2)

The Variance of $\begin{aligned}(4.5) & =\left(\frac{p_{3}}{\left(1-p_{2}\right)}\right)^{n}\left(\frac{p_{1}}{\left(1-p_{2}\right)}\right)^{x} \frac{1}{\left(\begin{array}{c}n+x-1 \\ x\end{array}\right)} \\ & \times{ }_{3} F_{2}\left(x+1, n, n ; x+n, 1 ; \frac{p_{1}}{\left(1-p_{2}\right)}\right)-\left(p_{1}^{2 x}\left(1-p_{2}\right)^{-2 x}\right)\end{aligned}$

The Variance of $(4.6)=\left(\frac{p_{3}}{\left(1-p_{1}\right)}\right)^{n}\left(\frac{p_{2}}{\left(1-p_{1}\right)}\right)^{y} \frac{1}{\left(\begin{array}{c}n+y-1 \\ y\end{array}\right)}$

$$
\times{ }_{3} F_{2}\left(y+1, n, n ; y+n, 1 ; \frac{p_{2}}{\left(1-p_{1}\right)}\right)-\left(p_{2}^{2 y}\left(1-p_{1}\right)^{-2 y}\right)
$$

Variance of $(4.7)=V(A+B+C-1)$

$$
=V(A)+V(B)+V(C)+2 \operatorname{cov}(A B)+2 \operatorname{cov}(B C)+2 \operatorname{cov}(A C)
$$


where

$$
\begin{gathered}
A=\frac{\left(\begin{array}{l}
s_{1} \\
x
\end{array}\right)}{\left(\begin{array}{c}
s_{1}+n-1 \\
x
\end{array}\right)} \\
B=\frac{\left(\begin{array}{c}
s_{2} \\
y
\end{array}\right)}{\left(\begin{array}{c}
s_{2}+n-1 \\
y
\end{array}\right)} \\
C=\sum_{r=0}^{x-1} \sum_{s=0} \frac{\left(\begin{array}{c}
r+s \\
r
\end{array}\right)\left(\begin{array}{c}
s_{1}+n-r-2 \\
s_{1}-r
\end{array}\right)\left(\begin{array}{c}
s_{1}+s_{2}+n-r-s-2 \\
s_{2}-s
\end{array}\right)}{\left(\begin{array}{c}
s_{1}+n-1 \\
s_{1}
\end{array}\right)\left(\begin{array}{c}
s_{1}+s_{2}+n-1 \\
s_{2}
\end{array}\right)}-1
\end{gathered}
$$

To obtain (4.10) we require $E\left(C^{2}\right), \mathrm{E}(\mathrm{AB}), \mathrm{E}(\mathrm{AC}), \mathrm{E}(\mathrm{BC})$

$$
\begin{aligned}
E\left(C^{2}\right) & =\sum_{r=0}^{x-1} \sum_{s=0}^{y-1} \frac{\left(\begin{array}{c}
r+s \\
r
\end{array}\right)}{\left(\begin{array}{c}
r+s+n-1 \\
n-1
\end{array}\right)} p_{1}^{r} p_{2}^{s} p_{3}^{n} \\
& \times F\left(\left((n-1)_{a+b}\right)^{2},(r+1)_{a},(s+1)_{b} ;(r+s+n)_{a+b},(1)_{a},(1)_{b} ; p_{1}, p_{2}\right) \\
& +2 \sum_{r=0}^{x-1} \sum_{s=0}^{y-1} \sum_{k=0}^{x-1} \sum_{l=0}^{y-1} \frac{\left(\begin{array}{c}
(k+l) \\
l
\end{array}\right)\left(\begin{array}{c}
r+s+n-k-l-2 \\
s-l
\end{array}\right)\left(\begin{array}{c}
r+n-k-2 \\
r-k
\end{array}\right)}{\left(\begin{array}{c}
r+s+n-1 \\
s
\end{array}\right)} p_{1}^{r} p_{2}^{s} p_{3}^{n} \\
& \times F\left((\beta)_{a+b},(n-1)_{a+b},(s+1)_{b},(\gamma)_{a},(r+1)_{a} ;(r+s+n)_{a+b},(\delta)_{b},(\gamma)_{a},(\theta)_{a} ; p_{1}, p_{2}\right)
\end{aligned}
$$

where $(r, s) \neq(k, l)$ and $s_{1}-r=a$ and $s_{2}-s=b$ and

$$
\begin{gathered}
\beta=(r+s+n-k-l-1), \quad \gamma=r+n-k-1, \quad \delta=s-l+1, \quad \theta=r-k+1 \\
E(A B)=\frac{\left(\begin{array}{c}
x+y+n-1 \\
y
\end{array}\right)}{\left(\begin{array}{c}
y+n-1 \\
y
\end{array}\right)} p_{1}^{x} p_{2}^{y} p_{3}^{n} F\left((x+y+n)_{a+b},(x+1)_{a},(n)_{b} ;(x+n)_{a},(y+n)_{b} ; p_{1}, p_{2}\right)
\end{gathered}
$$




$$
\begin{aligned}
& E(A C)=\sum_{r=0}^{x-1} \sum_{s=0}^{y-1} \frac{\left(\begin{array}{c}
r+s \\
r
\end{array}\right)\left(\begin{array}{l}
r \\
x
\end{array}\right)}{\left(\begin{array}{c}
r+n-1 \\
x
\end{array}\right)} \frac{p_{1}^{r} p_{2}^{s} p_{3}^{n}}{\left(1-p_{2}\right)^{n-1}} \\
& \times{ }_{3} F_{2}\left((r+1),(r+n-x) ;(r-x+1),(r+n) ; \frac{p_{1}}{\left(1-p_{2}\right)}\right) \\
& E(B C)= \sum_{r=0}^{x-1} \sum_{s=0}^{y-1} \frac{\left(\begin{array}{c}
r+s \\
r
\end{array}\right)\left(\begin{array}{c}
s \\
y
\end{array}\right)}{\left(\begin{array}{c}
s+n-1 \\
y
\end{array}\right)} \frac{p_{1}^{r} p_{2}^{s} p_{3}^{n}}{\left(1-p_{1}\right)^{n-1}} \\
& \quad \times{ }_{3} F_{2}\left((s+1),(s+n-y),(n-1) ;(s-y+1),(s+n) ; \frac{p_{2}}{\left(1-p_{1}\right)}\right)
\end{aligned}
$$

$$
\begin{aligned}
& \text { The MLE of }(4.1)=\left(\frac{s_{1}}{\left(n+s_{1}\right)}\right)^{x} \\
& \text { The MLE of }(4.2)=\left(\frac{s_{2}}{\left(n+s_{2}\right)}\right)^{y}
\end{aligned}
$$

The MLE of $(4.3)=\left(\frac{s_{1}}{\left(n+s_{1}\right)}\right)^{x}+\left(\frac{s_{2}}{\left(n+s_{2}\right)}\right)^{y}+\sum_{r=0}^{x-1} \sum_{s=0}^{y-1}\left(\begin{array}{c}r+s \\ r\end{array}\right) \frac{s_{1}^{r} s_{2}^{s} n}{\left(n+s_{1}+s_{2}\right)^{r+s+1}}-$

$$
\text { Variance of } \begin{aligned}
(4.15) & =\frac{n}{(n+1)^{2 x}}\left(\frac{p_{1}}{1-p_{2}}\right)\left(\frac{p_{3}}{1-p_{2}}\right)^{n} \\
& \times{ }_{4 x} F_{4 x-1}\left((n+1)^{2 x+1},(2)^{2 x-1} ;(n+2)^{2 x},(1)^{2 x-1} ; \frac{p_{1}}{1-p_{2}}\right) \\
& -\frac{n^{2}}{(n+1)^{2 x}}\left(\frac{p_{1}}{1-p_{2}}\right)^{2}\left(\frac{p_{3}}{1-p_{2}}\right)^{2 n} \\
& \times\left({ }_{2 x} F_{2 x-1}\left((n+1)^{x+1},(2)^{x-1} ;(n+2)^{x},(1)^{x-1} ; \frac{p_{1}}{1-p_{2}}\right)\right)^{2}
\end{aligned}
$$

Variance of $(4.16)=\frac{n}{(n+1)^{2 y}}\left(\frac{p_{2}}{1-p_{1}}\right)\left(\frac{p_{3}}{1-p_{1}}\right)^{n}$

$$
\begin{aligned}
& \times{ }_{4 y} F_{4 y-1}\left((n+1)^{2 y+1},(2)^{2 y-1} ;(n+2)^{2 y},(1)^{2 y-1} ; \frac{p_{2}}{1-p_{1}}\right) \\
& -\frac{n^{2}}{(n+1)^{2 y}}\left(\frac{p_{2}}{1-p_{1}}\right)^{2}\left(\frac{p_{3}}{1-p_{1}}\right)^{2 n} \\
& \times\left(2 y F_{2 y-1}\left((n+1)^{y+1},(2)^{y-1} ;(n+2)^{y},(1)^{y-1} ; \frac{p_{2}}{1-p_{1}}\right)\right)^{2}
\end{aligned}
$$




$$
\begin{aligned}
\text { Variance of }(4.17) & =V\left(A^{\prime}+B^{\prime}+C^{\prime}-1\right) \\
& =V\left(A^{\prime}\right)+V\left(B^{\prime}\right)+V\left(C^{\prime}\right)+2 \operatorname{cov}\left(A^{\prime} B^{\prime}\right)+2 \operatorname{cov}\left(B^{\prime} C^{\prime}\right)+2 \operatorname{cov}\left(A^{\prime} C^{\prime}\right)
\end{aligned}
$$

where

$$
\begin{gathered}
A^{\prime}=\left(\frac{s_{1}}{\left(n+s_{1}\right)}\right)^{x} \\
B^{\prime}=\left(\frac{s_{2}}{\left(n+s_{2}\right)}\right)^{y} \\
C^{\prime}=\sum_{r=0}^{x-1} \sum_{s=0}^{y-1}\left(\begin{array}{c}
r+s \\
r
\end{array}\right) \frac{s_{1}^{r} s_{2}^{s} n}{\left(n+s_{1}+s_{1}\right)^{r+s+1}}-1
\end{gathered}
$$

To obtain (4.20) we require $\mathrm{E}\left(\mathrm{C}^{\prime}\right), E\left(C^{\prime 2}\right), \mathrm{E}\left(\mathrm{A}^{\prime} \mathrm{B}^{\prime}\right), \mathrm{E}\left(\mathrm{A}^{\prime} \mathrm{C}^{\prime}\right), \mathrm{E}\left(\mathrm{B}^{\prime} \mathrm{C}^{\prime}\right)$

$$
\begin{aligned}
E\left(C^{\prime}\right) & =p_{3}^{n} F\left(\left((n)_{\left(s_{1}+s_{2}\right)}\right)^{2} ;(n+1)_{\left(s_{1}+s_{2}\right)} ; p_{1}, p_{2}\right) \\
& +\sum_{s=1}^{y-1} \frac{n^{2} p_{2} p_{3}^{n}}{(n+1)^{s+1}} F\left(\left((n+1)_{\xi}\right)^{s+2},\left[(2)_{s_{2}-1}\right]^{s-1} ;\left[(n+2)_{\xi}\right]^{s+1},\left[(1)_{s_{2}-1}\right]^{s-1} ; p_{1}, p_{2}\right) \\
& +\sum_{r=1}^{x-1} \frac{n^{2} p_{1} p_{3}^{n}}{(n+1)^{r+1}} F\left(\left[(n+1)_{\alpha}\right]^{r+2},\left[(2)_{s_{1}-1}\right]^{r-1} ;\left[(n+2)_{\alpha}\right]^{r+1},\left[(1)_{s_{1}-1}\right]^{r-1} ; p_{1}, p_{2}\right) \\
& +\sum_{r=1}^{x-1} \sum_{s=1}^{y-1}\left(\begin{array}{c}
r+s \\
r
\end{array}\right) p_{1} p_{2} p_{3}^{n} \frac{n^{2}(n+1)}{(n+2)^{r+s+1}} \\
& \times F\left(\left[(n+2)_{\lambda}\right]^{r+s+2},\left[(2)_{s_{1}-1}\right]^{r-1},\left[(2)_{s_{2}-1}\right]^{s-1} ;\left[(n+3)_{\lambda}\right]^{r+s+1},\left[(1)_{s_{1}-1}\right]^{r-1},\left[(1)_{s_{2}-1}\right]^{s-1} ; p_{1}, p_{2}\right)
\end{aligned}
$$

where

$$
\lambda=\left(s_{1}-1\right)+\left(s_{2}-1\right), \alpha=\left(s_{1}-1\right)+s_{2}, \quad \xi=s_{1}+\left(s_{2}-1\right)
$$




$$
\begin{aligned}
& E\left(\left(C^{\prime}\right)^{2}\right)=p_{3}^{n} F\left(\left[(n)_{s_{1}+s_{2}}\right]^{3} ;\left((n+1)_{s_{1}+s_{2}}\right)^{2} ; p_{1}, p_{2}\right) \\
& +\sum_{s=1}^{y-1} \frac{n^{3} p_{2} p_{3}^{n}}{(n+1)^{2 s+2}} \\
& \times F\left(\left[(n+1)_{\xi}\right]^{2 s+3},\left[(2)_{s_{2}-1}\right]^{2 s-1} ;((n+2) \xi)^{2 s+2},\left[(1)_{s_{2}-1}\right]^{2 s-1} ; p_{1}, p_{2}\right) \\
& +\sum_{r=1}^{x-1} \frac{n^{3} p_{1} p_{3}^{n}}{(n+1)^{2 r+2}} \\
& \times F\left(\left[(n+1)_{\alpha}\right]^{2 r+3},\left[(2)_{s_{1}-1}\right]^{2 r-1} ;\left(\left[(n+2)_{\alpha}\right)^{2 r+2}\right],\left[(1)_{s_{1}-1}\right]^{2 r-1} ; p_{1}, p_{2}\right) \\
& +\sum_{r=1}^{x-1} \sum_{s=1}^{y-1}\left(\left(\begin{array}{c}
r+s \\
r
\end{array}\right)\right)^{2} p_{1} p_{2} p_{3}^{n} \frac{n^{2}(n+1)}{(n+2)^{2 r+2 s+2}} \\
& \times F\left(\left[(n+2)_{\lambda}\right]^{\tau+1},\left[(2)_{s_{1}-1}\right]^{2 r-1},\left[(2)_{s_{2}-1}\right]^{2 s-1} ;\left[(n+3)_{\lambda}\right]^{\tau},\left[(1)_{s_{1}-1}\right]^{2 r-1},\left[(1)_{s_{2}-1}\right]^{2 s-1} ; p_{1}, p_{2}\right) \\
& +2 \sum_{r=1}^{x-1} \sum_{s=1}^{y-1} \sum_{k=1}^{x-1} \sum_{l=1}^{y-1}\left(\left(\begin{array}{c}
r+s \\
r
\end{array}\right)\right)\left(\left(\begin{array}{c}
k+l \\
k
\end{array}\right)\right) p_{1} p_{2} p_{3}^{n} \frac{n^{3}(n+1)}{(n+2)^{r+s+k+l+2}} \\
& F\left(\left[(n+2)_{\lambda}\right]^{\mu+3},\left[(2)_{s_{1}-1}\right]^{\zeta},\left[(2)_{s_{2}-1}\right]^{\sigma} ;\left[(n+3)_{\lambda}\right]^{\mu+2},\left[(1)_{s_{1}-1}\right]^{\zeta},\left[(1)_{s_{2}-1}\right]^{\sigma} ; p_{1}, p_{2}\right) \\
& +2 \sum_{r=0}^{x-1} \sum_{k=0}^{x-1} p_{1} p_{3}^{n} \frac{n^{3}}{(n+1)^{r+k+2}} \\
& F\left(\left[(n+1)_{\left(s_{1}-1\right)+s_{2}}\right]^{r+k+3},\left[(2)_{s_{1}-1}\right]^{\zeta} ;\left[(n+2)_{\left(s_{1}-1\right)+s_{2}}\right]^{r+k+2},\left[(1)_{s_{1}-1}\right]^{\zeta}, ; p_{1}, p_{2}\right) \\
& +2 \sum_{s=0}^{y-1} \sum_{l=0}^{y-1} p_{2} p_{3}^{n} \frac{n^{3}}{(n+1)^{s+l+2}} \\
& F\left(\left[(n+1)_{\xi}\right]^{s+l+3},\left[(2)_{s_{2}-1}\right]^{\sigma} ;\left[(n+2)_{\xi}\right]^{s+l+2},\left[(1)_{s_{2}-1}\right]^{\sigma}, ; p_{1}, p_{2}\right)
\end{aligned}
$$

where

$$
\lambda=\left(s_{1}-1\right)+\left(s_{2}-1\right), \tau=2 r+2 s+2, \mu=r+s+k+l, \zeta=r+k-1, \sigma=s+l-1
$$

and $(r, s) \neq(k, l)$

$$
\begin{aligned}
E\left(A^{\prime} B^{\prime}\right) & =\frac{n}{\phi^{(x+y-1)}} p_{1} p_{2} p_{3}^{n} \\
& \times F\left((\phi+1)_{\lambda}\left[\phi_{\psi}\right]^{x},\left[(2)_{\psi}\right]^{x-1},\left[(1)_{\chi}\right]^{y-1} ;\left[(\phi+1)_{\psi}\right]^{x},\left[(\phi+1)_{\chi}\right]^{y},\left[(1)_{\psi}\right]^{x-1},\left[(1)_{\chi}\right]^{y-1} ; p_{1}, p_{2}\right)
\end{aligned}
$$

where

$$
\psi=s_{1}-1, \chi=s_{2}-1, \phi=n+1
$$




$$
\begin{aligned}
E\left(A^{\prime} C^{\prime}\right) & =\sum_{r=0}^{x-1} \frac{n^{2}}{(n+1)^{r+x+1}} p_{1} p_{3}^{n} \\
& \times F\left(\left[(\phi)_{\alpha}\right]^{r+2},\left[(n+1)_{\psi}\right]^{x},\left[(2)_{\psi}\right]^{x+r-1} ;\left[(n+2)_{\alpha}\right]^{r+1},\left[(n+2)_{\psi}\right]^{x},\left[(1)_{\psi}\right]^{x+r-1} ; p_{1}, p_{2}\right) \\
& +\sum_{r=0}^{x-1} \sum_{s=1}^{y-1}\left(\begin{array}{c}
r+s \\
r
\end{array}\right) \frac{n^{2}}{(n+1)^{x-1}(n+2)^{\omega}} p_{1} p_{2} p_{3}^{n} \\
& \times F\left((n+2)_{\lambda}^{\omega+1}\left[(\phi)_{\psi}\right]^{x},\left[(2)_{\psi}\right]^{\varphi},\left[(2)_{\chi}\right]^{s-1} ;\left[(n+3)_{\lambda}\right]^{\omega},\left[(n+2)_{\psi}\right]^{x},\left[(1)_{\psi}\right]^{\varphi},\left[(1)_{\chi}\right]^{s-1} ; p_{1}, p_{2}\right)
\end{aligned}
$$

where

$$
\begin{aligned}
& \varphi=x+r-1, \omega=r+s+1, \Omega=s+y-1 \\
E\left(B^{\prime} C^{\prime}\right)= & \sum_{s=0}^{y-1} \frac{n^{2}}{(n+1)^{y+s+1}} p_{2} p_{3}^{n} \\
\times & F\left(\left[(\phi)_{\xi))}\right]^{s+2},\left[(\phi)_{\chi}\right]^{y},\left[(2)_{\chi}\right]^{\Omega} ;\left[(n+2)_{\xi}\right]^{s+1},\left[(n+2)_{\chi}\right]^{y},\left[(1)_{\chi}\right]^{\Omega} ; p_{1}, p_{2}\right) \\
+ & \sum_{r=1}^{x-1} \sum_{s=0}^{y-1}\left(\begin{array}{c}
r+s \\
r
\end{array}\right) \frac{n^{2}}{(n+1)^{y-1}(n+2)^{\omega}} p_{1} p_{2} p_{3}^{n} \\
& \times F\left((n+2)_{\lambda}^{\omega+1}\left[(\phi)_{\chi}\right]^{y},\left[(2)_{\chi}\right]^{\Omega},\left[(2)_{\psi}\right]^{r-1} ;\left[(n+3)_{\lambda}\right]^{\omega},\left[(n+2)_{\chi}\right]^{y},\left[(1)_{\chi}\right]^{\Omega},\left[(1)_{\psi}\right]^{r-1} ; p_{1}, p_{2}\right)
\end{aligned}
$$

\section{Conclusion : Comparision of UMVUE and MLE}

In order to get an idea of the efficiency of the two types of estimation,MLE and UMVUE, we have calculated the MSE of both the estimators for

1. Estimation of $p_{1}^{r_{1}} p_{2}^{r_{2}} p_{3}^{r_{3}}$ for various values of $r_{1}, r_{2}$ and $r_{3}$.

We have depicted the same with graphs (Fig1and 2) done below for $p_{1}^{r_{1}} p_{2}^{r_{2}} p_{3}^{r_{3}}$ when $r_{1}=1, r_{2}=0$ and $r_{3}=0$ for $p_{1}$ values ranging from 0.1 to 0.8 .

2. Estimation of reliability function $\mathrm{R}(\mathrm{x}, \mathrm{y})$ for different values of $\mathrm{X}$ and $\mathrm{Y}$ such as $\mathrm{X}, \mathrm{Y}=1,2$ and for $p_{1}, p_{2}$ and $p_{3}$ values ranging between 0.1 and 0.8 .

From the graphs of MSE of MLE and UMVUE of $p_{1}$ and of reliability function for various cases drawn in Figs 1-10 we observe that for some cases UMVUE is more efficient and for some cases MLE is more efficient. It is also observed that as n increases MSE of UMVUE and MLE tends to 0 for all cases. Hence we can conjecture that the UMVUE and MLE estimates are consistent.

Further Fig 1 and 2 compares the MSE of UMVUE and MLE of $p_{1}$. When the value of $p_{1}$ is $\geq 0.5$ the MSE of UMVUE of $p_{1}$ is less than the MSE of MLE of $p_{1}$. When $p_{1}$ values are $<0.5$ the MSE of UMVUE of $p_{1}$ is greater than the MSE of MLE of $p_{1}$. We have verified for the MSE of MLE and UMVUE of $p_{2}$ and $p_{3}$ for values of $p_{1}, p_{2}$ and $p_{3}$ ranging from 0.1 to 0.8 . The same pattern, as 
seen for $p_{1}$, is observed for $p_{2}$ also. However the reverse occurs for $p_{3}$. When the values of $p_{3}$ are $\geq 0.5$ the MSE of UMVUE of $p_{3}$ is greater than the MSE of MLE of $p_{3}$. When $p_{3}$ values $<0.5$ the MSE of UMVUE of $p_{3}$ is less than the MSE of MLE of $p_{3}$.

Thus we observe that in certain cases UMVUE is a better choice and in other cases MLE is better with respect to MSE of the estimator obtained. UMVUE is the best among the class of unbiased estimators. If it attains Cramer Rao (CR) lower bound, then it is the best estimator. In the above case UMVUE does not attain CR lower bound. Due to this it is quite likely that MLE might be a better estimator and hence there is a need to consider the MLE.

The graphs of MSE of MLE and UMVUE for reliability function are drawn in Figs 3-10. It is observed that in some cases UMVUE is more efficient and for some cases MLE is more efficient.For example when $p_{1}=0.5 ; p_{2}=0.4 ; p_{3}=0.1$ the UMVUE estimates are better than the MLE estimates. 


\section{Graphs for MSE of UMVUE and MLE for p1}
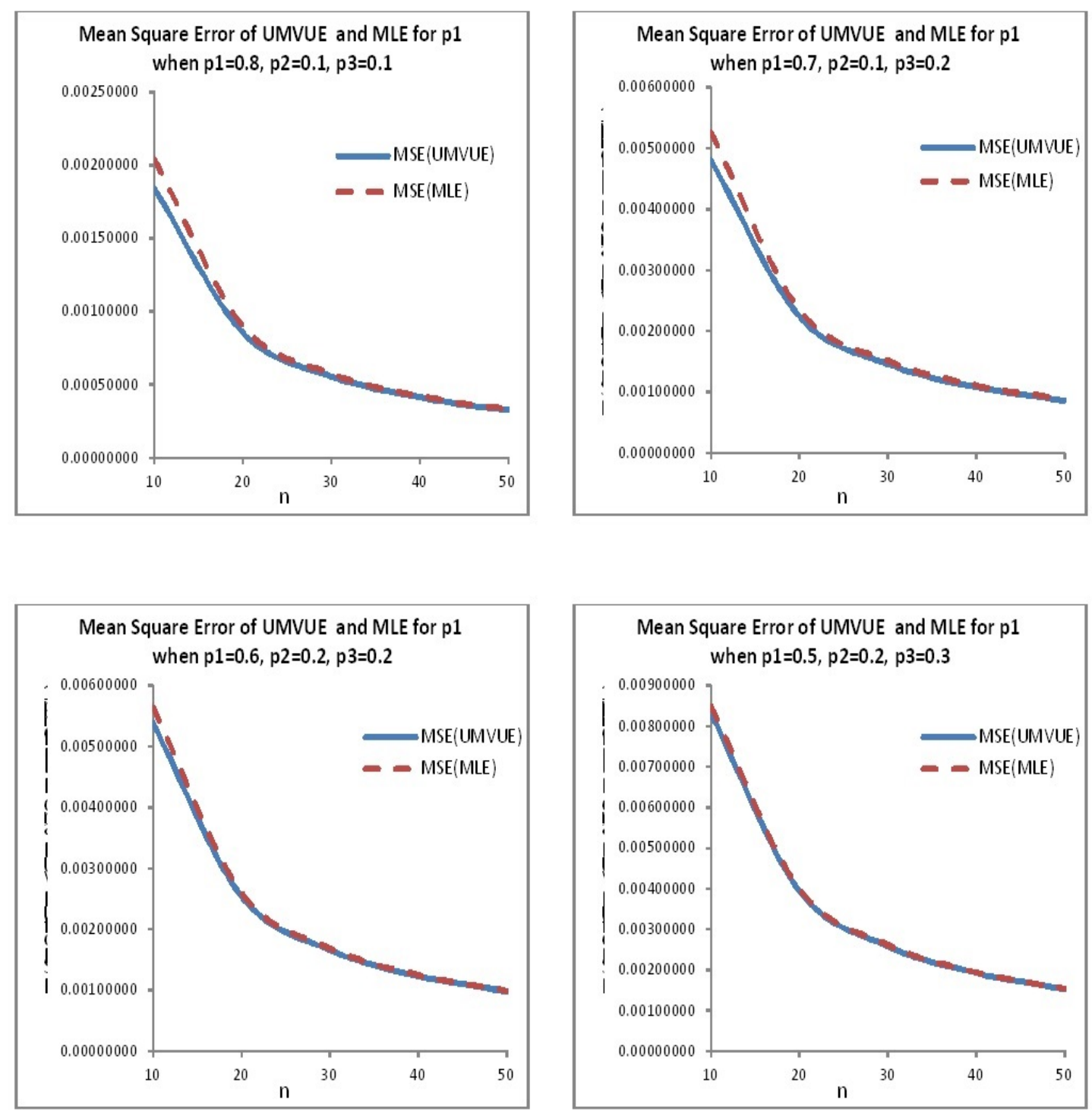

Fig. 1. 


\section{Graphs for MSE of UMVUE and MLE for $\mathrm{p1}$}
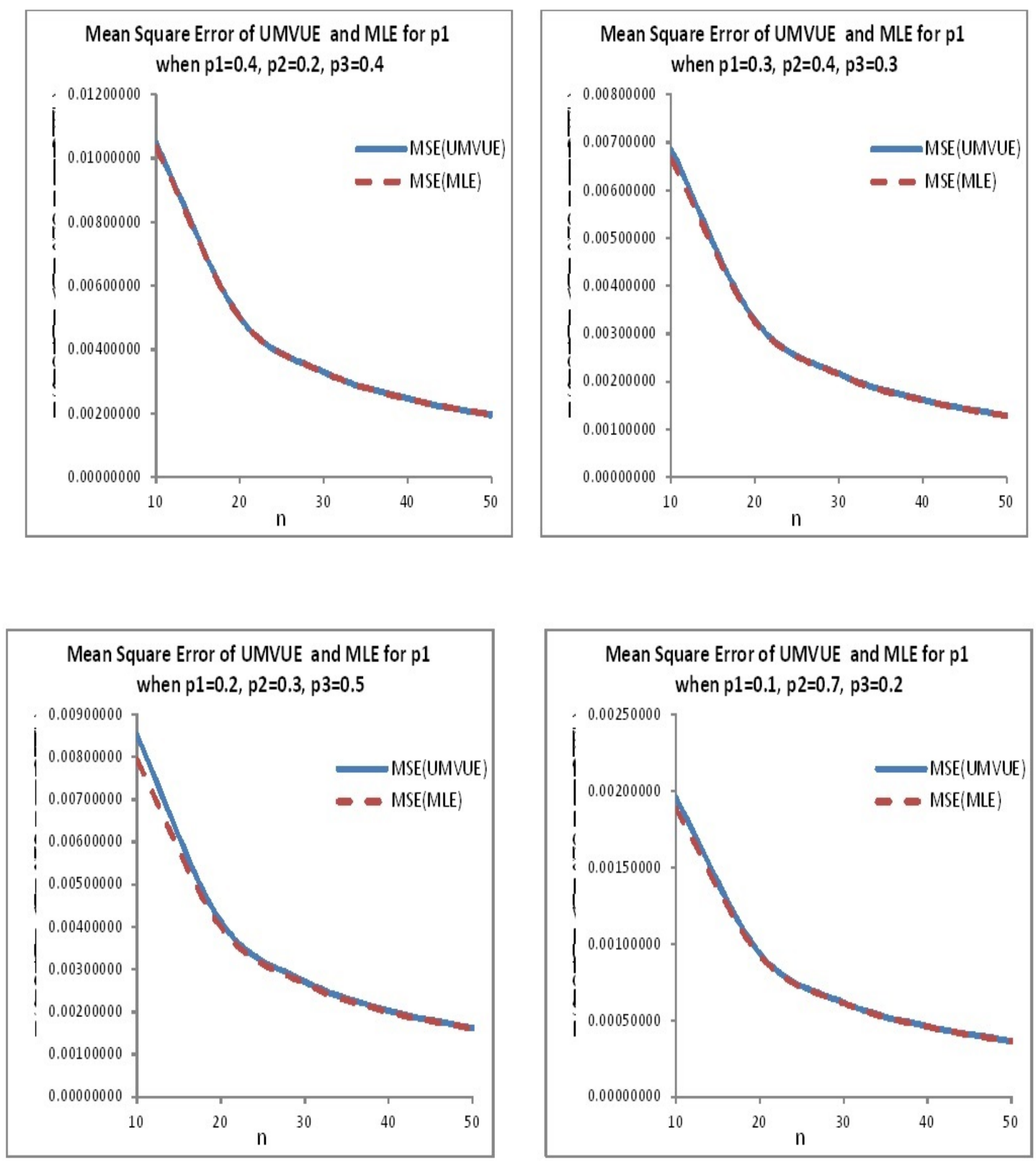

Fig. 2. 
Graphs for MSE of UMVUE and MLE for Reliability function

\section{CASE: $X=1 Y=1$}
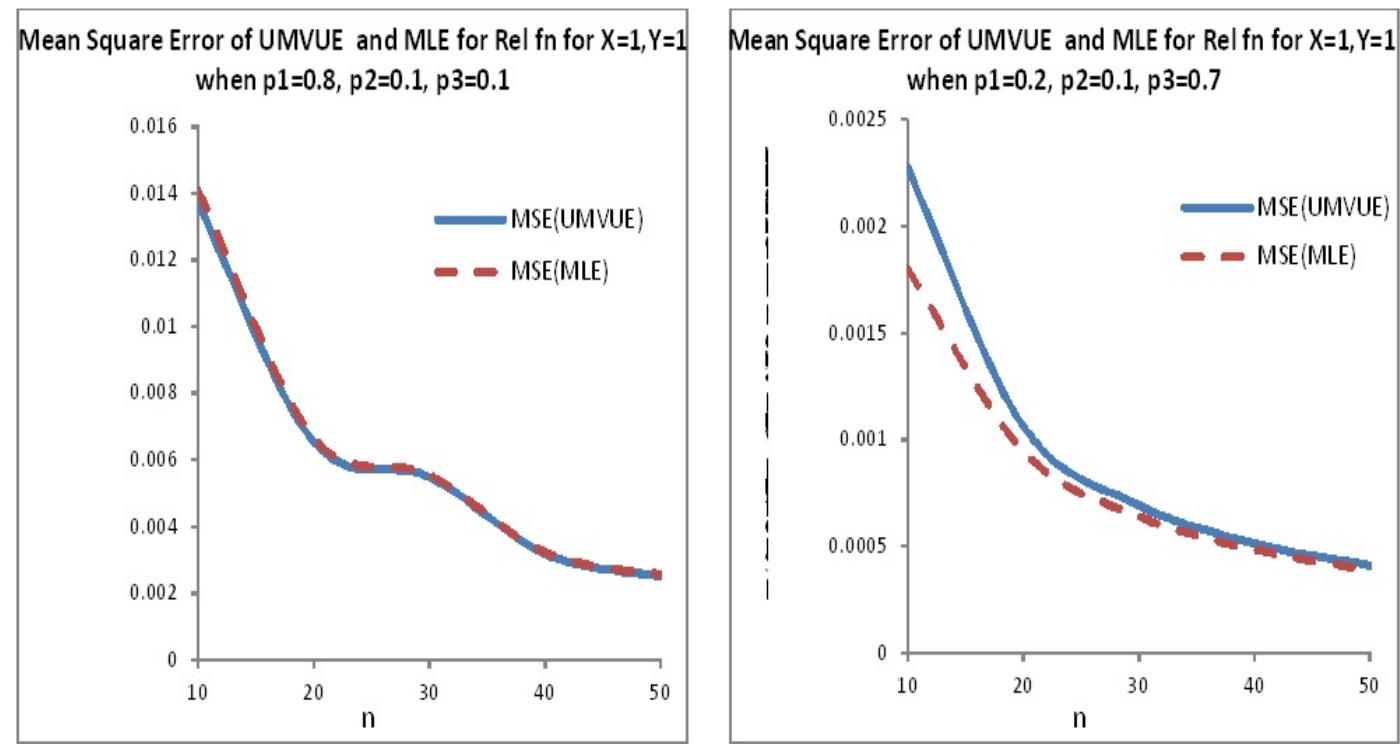

Mean Square Error of UMVUE and MLE forREL $f n X=1 Y=1$ when $\mathrm{p} 1=0.6, \mathrm{p} 2=0.2, \mathrm{p} 3=0.2$

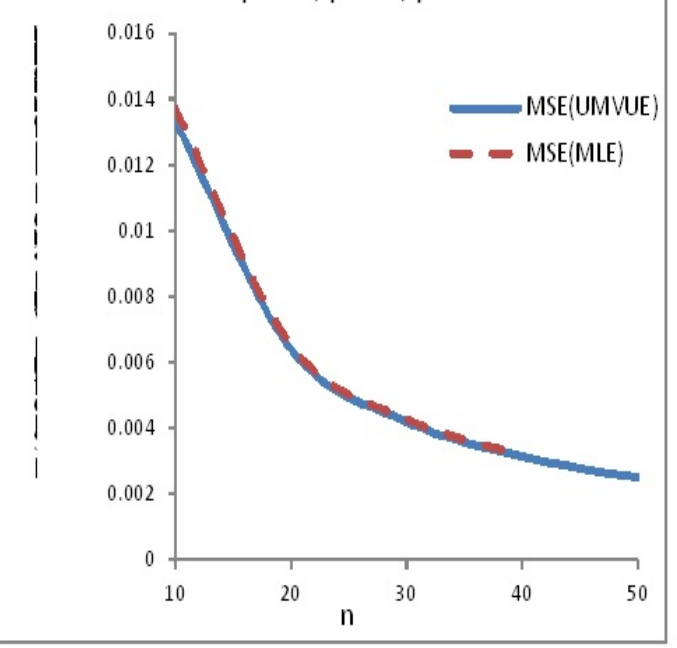

Mean Square Error of UMVUE and MLE forREL $f n X=1 Y=1$ when $\mathrm{p} 1=0.5, \mathrm{p} 2=0.4, \mathrm{p} 3=0.1$

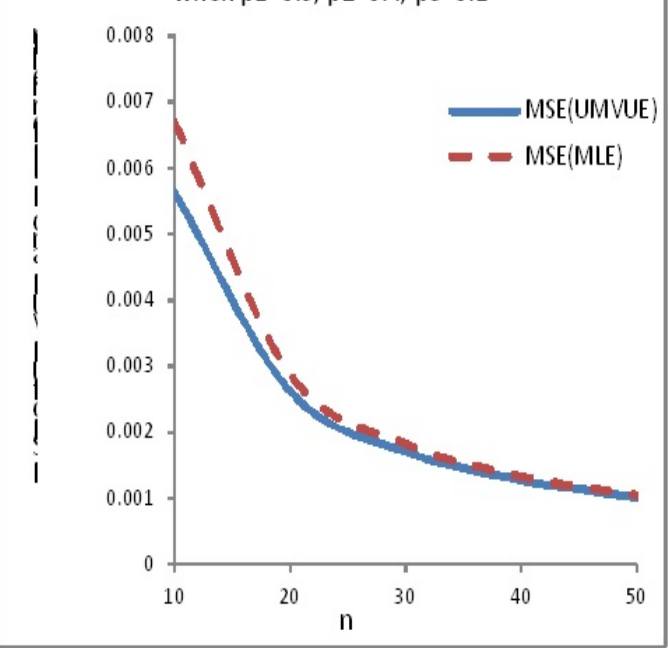

Fig. 3. 


\section{Graphs for MSE of UMVUE and MLE for Reliability function}

\section{CASE: $X=1 Y=1$}
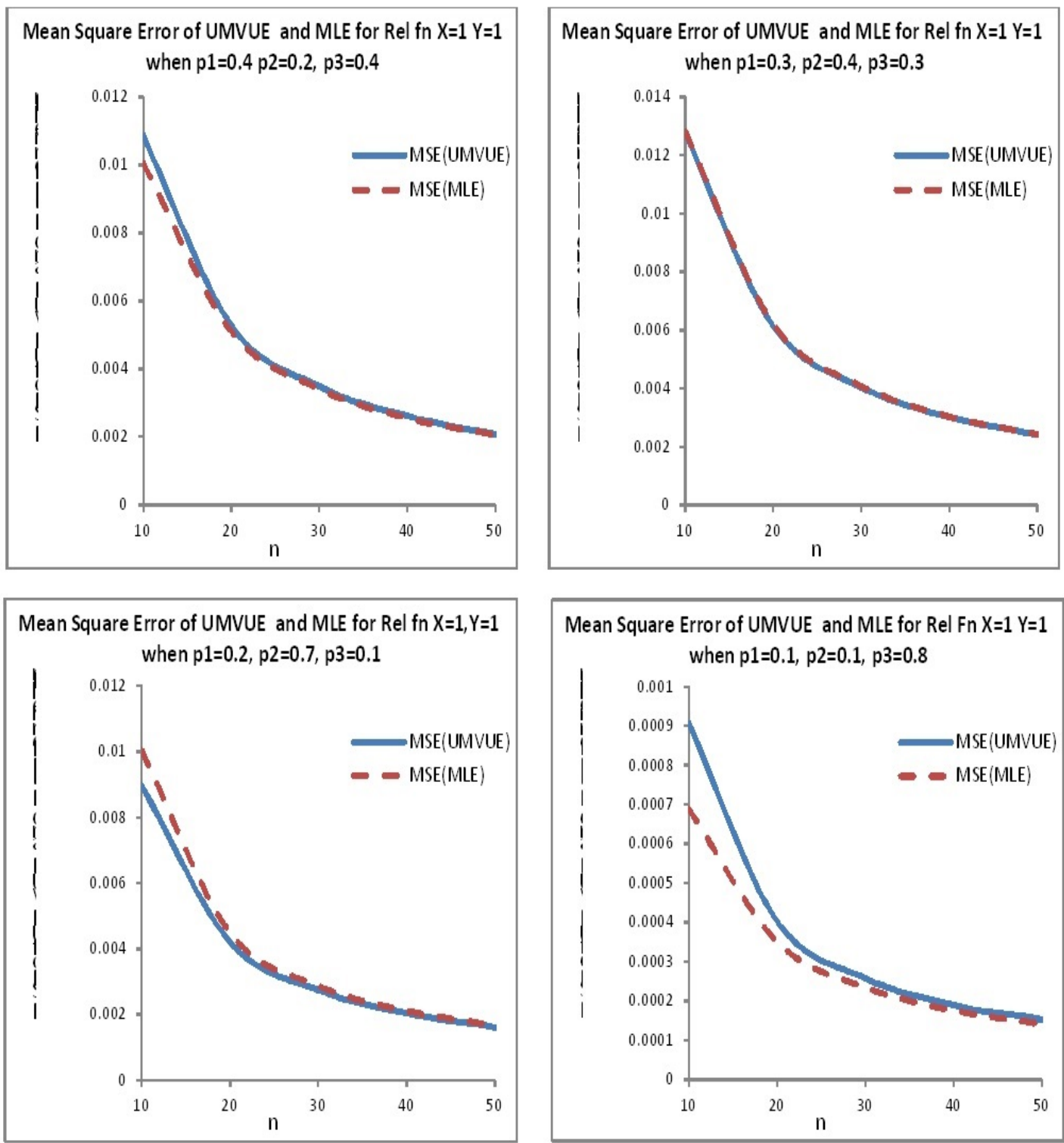

Fig. 4. 


\section{Graphs for MSE of UMVUE and MLE for Reliability function}

CASE: $X=1 Y=2$
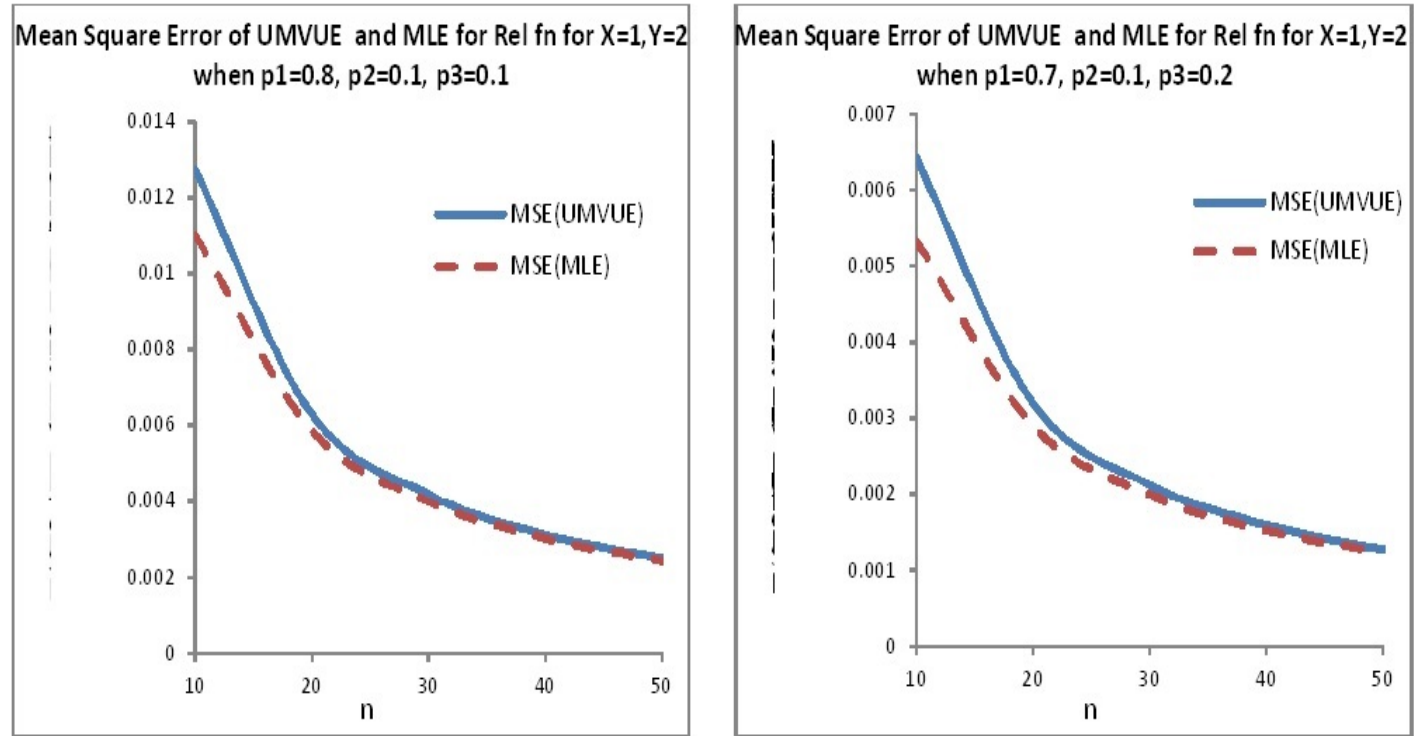

Mean Square Error of UMVUE and MLE forREL $f n X=1 Y=2$ when $\mathrm{p} 1=0.6, \mathrm{p} 2=0.2, \mathrm{p} 3=0.2$
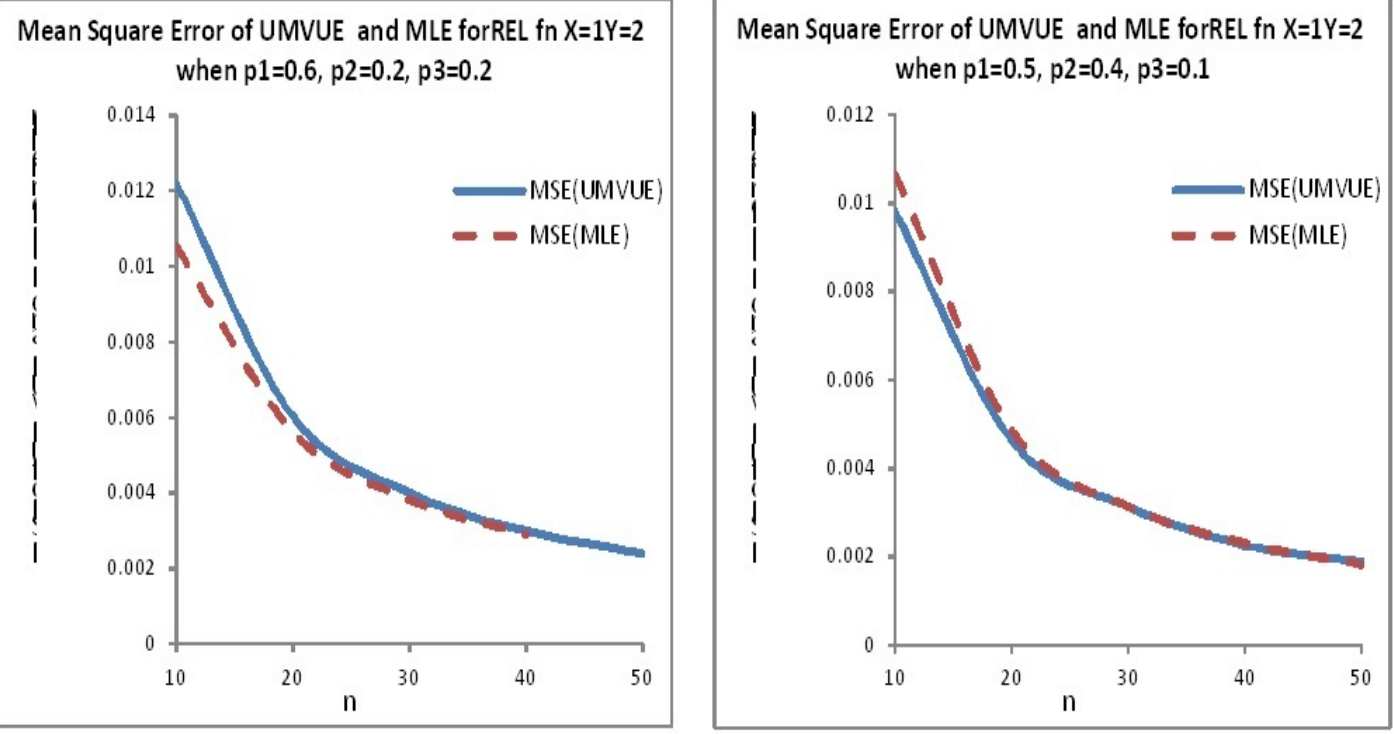

Fig. 5. 


\section{Graphs for MSE of UMVUE and MLE for Reliability function}

\section{CASE: $X=1 Y=2$}
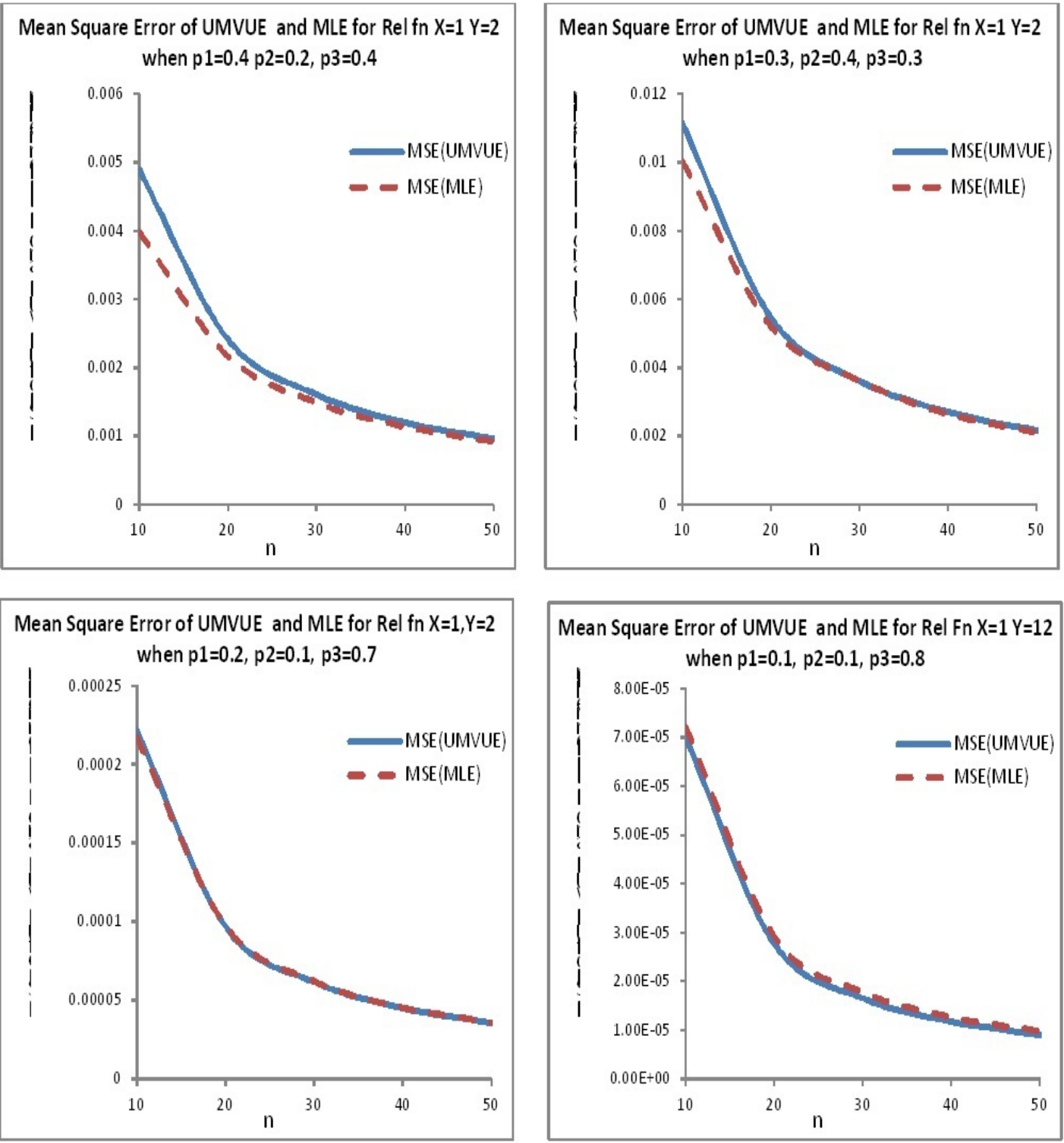

Fig. 6. 


\section{Graphs for MSE of UMVUE and MLE for Reliability function}

CASE: $X=2 Y=2$
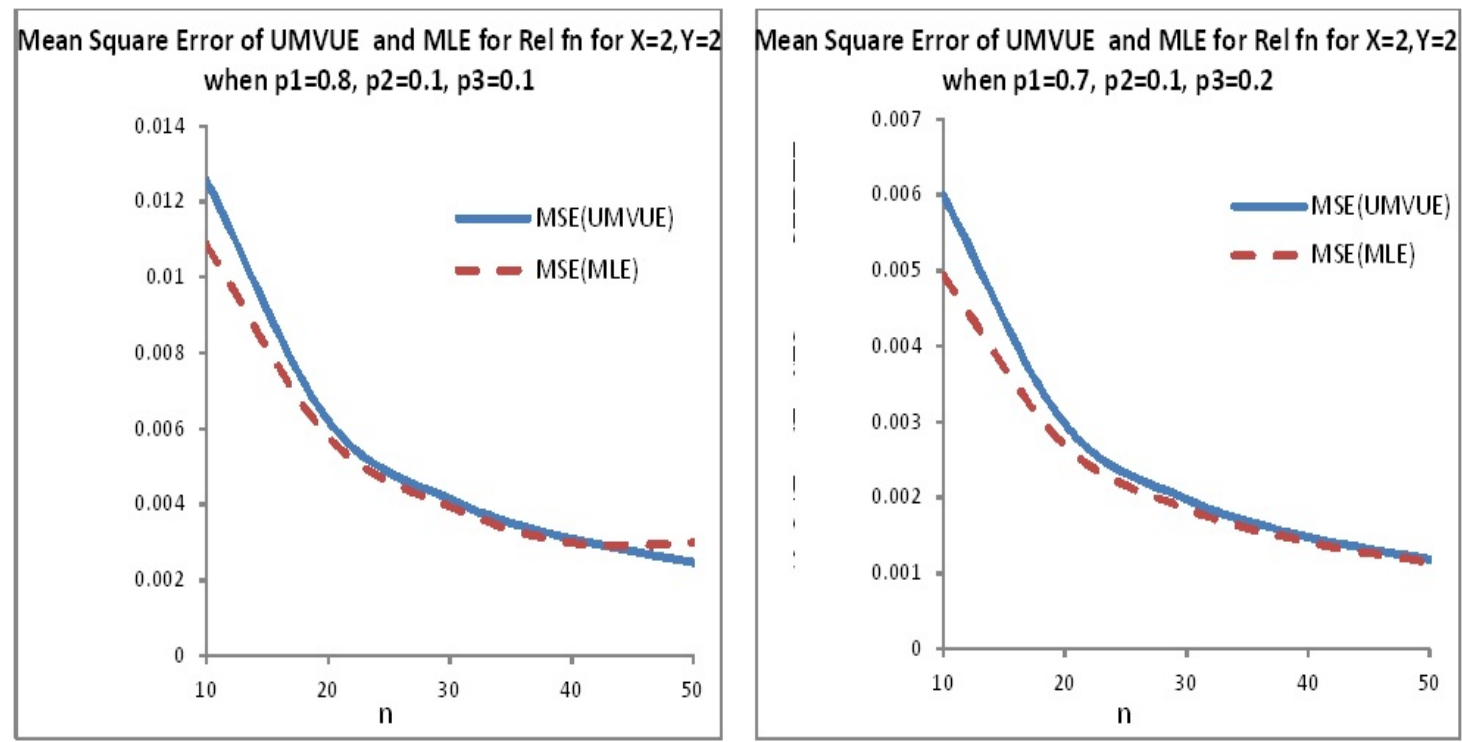

Mean Square Error of UMVUE and MLE forREL in $X=2, Y=2$ when $\mathrm{p} 1=0.6, \mathrm{p} 2=0.2, \mathrm{p} 3=0.2$
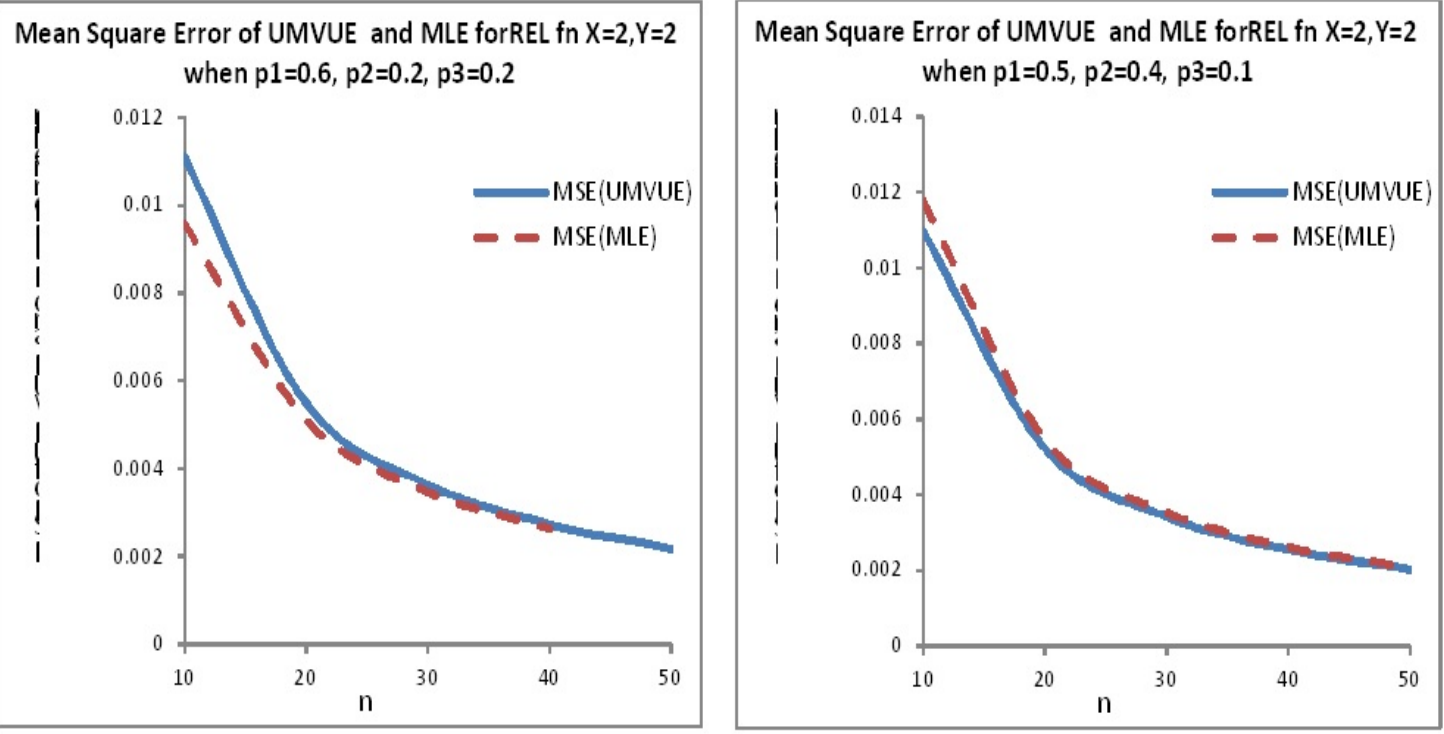

Fig. 7. 


\section{Graphs for MSE of UMVUE and MLE for Reliability function}

\section{CASE: $X=2 Y=2$}
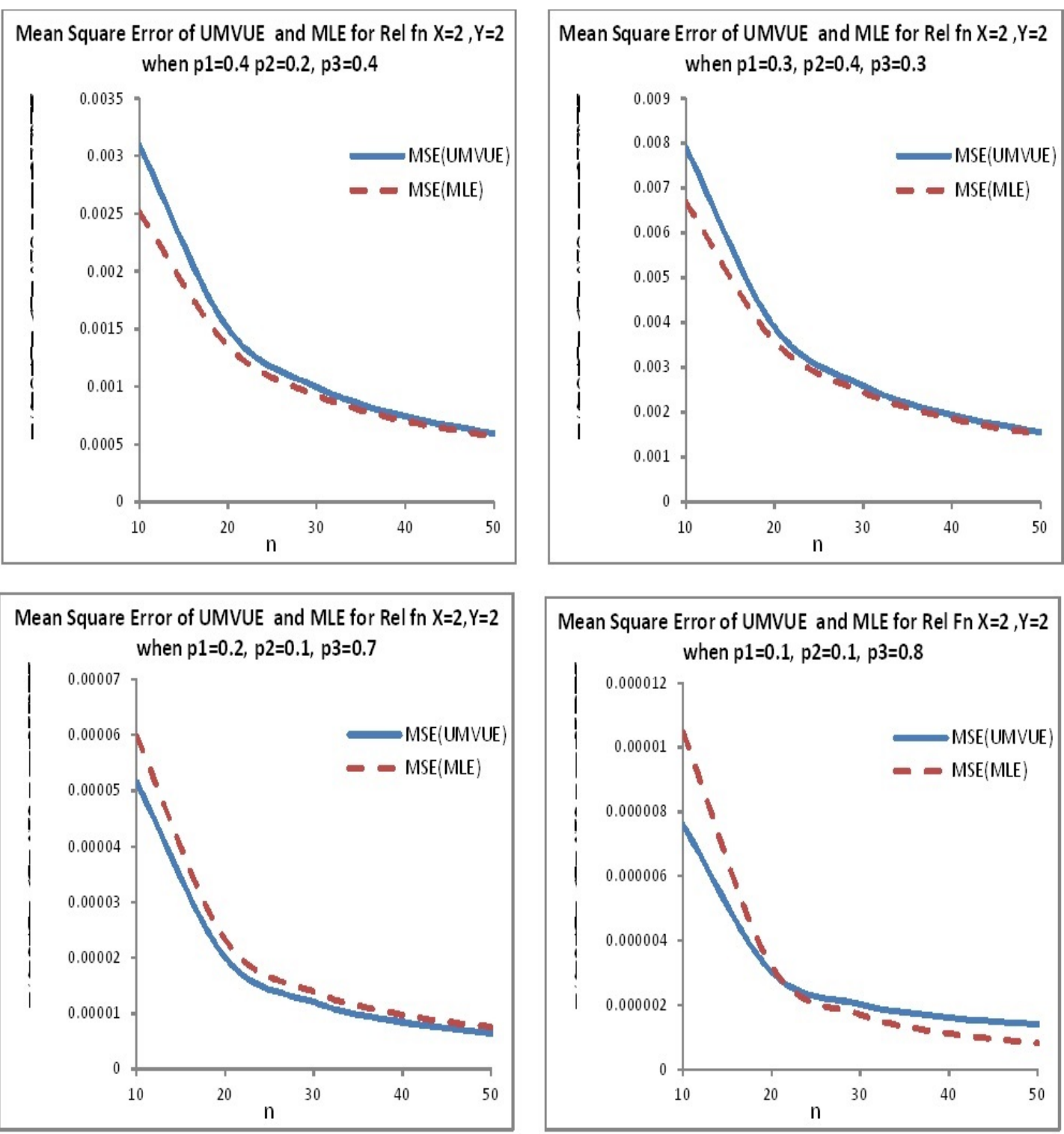

Fig. 8. 


\section{Graphs for MSE of UMVUE and MLE for Reliability function}

CASE: $X=2 Y=1$
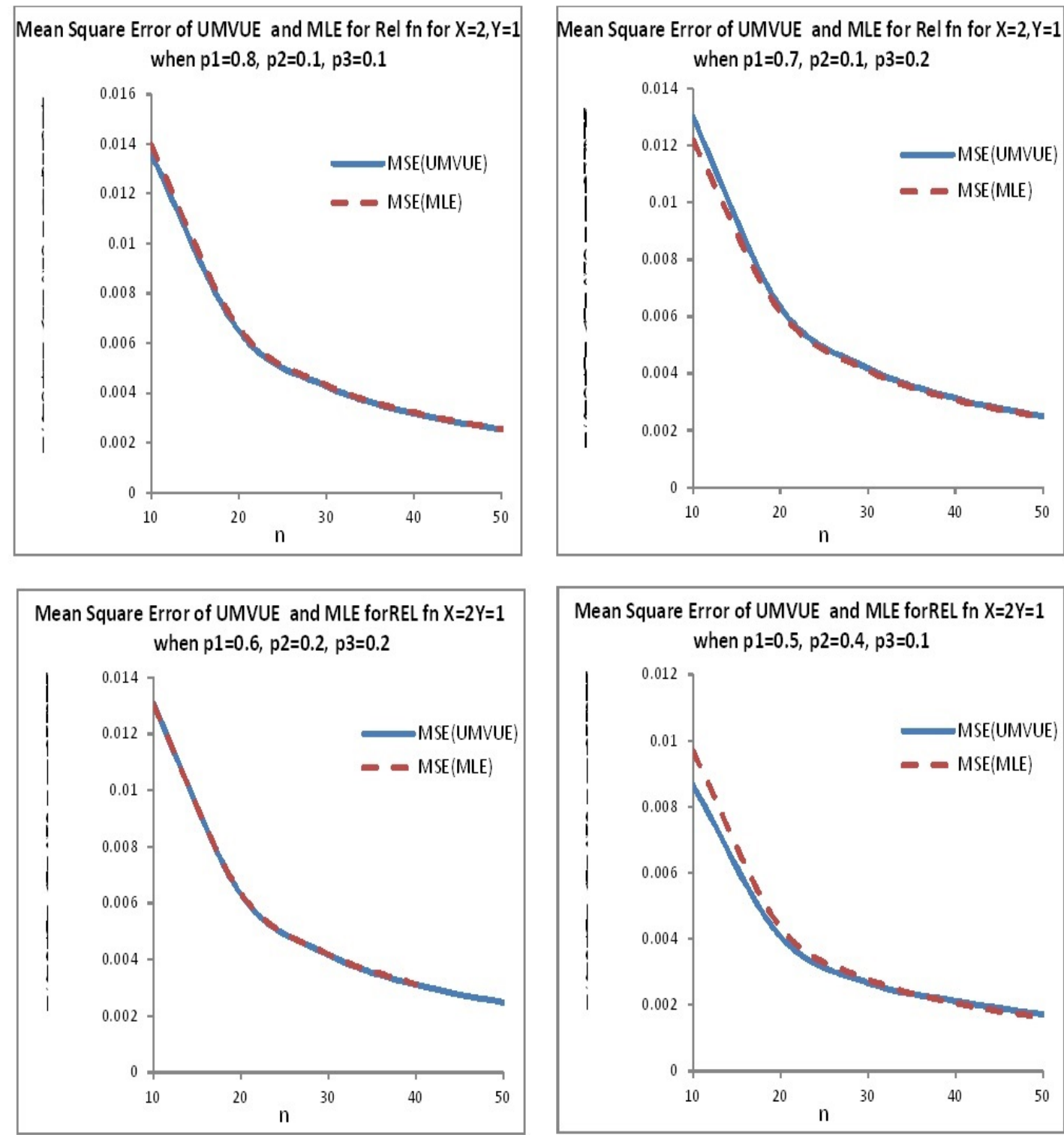

Mean Square Error of UMVUE and MLE forREL $f n X=2 Y=1$ when $\mathrm{p} 1=0.5, \mathrm{p} 2=0.4, \mathrm{p} 3=0.1$

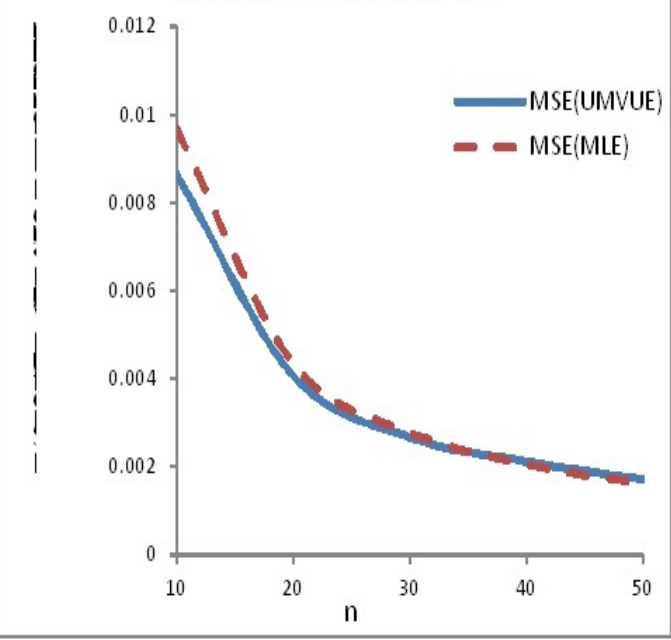

Fig. 9. 


\section{Graphs for MSE of UMVUE and MLE for Reliability function}

\section{CASE: $X=2 Y=1$}
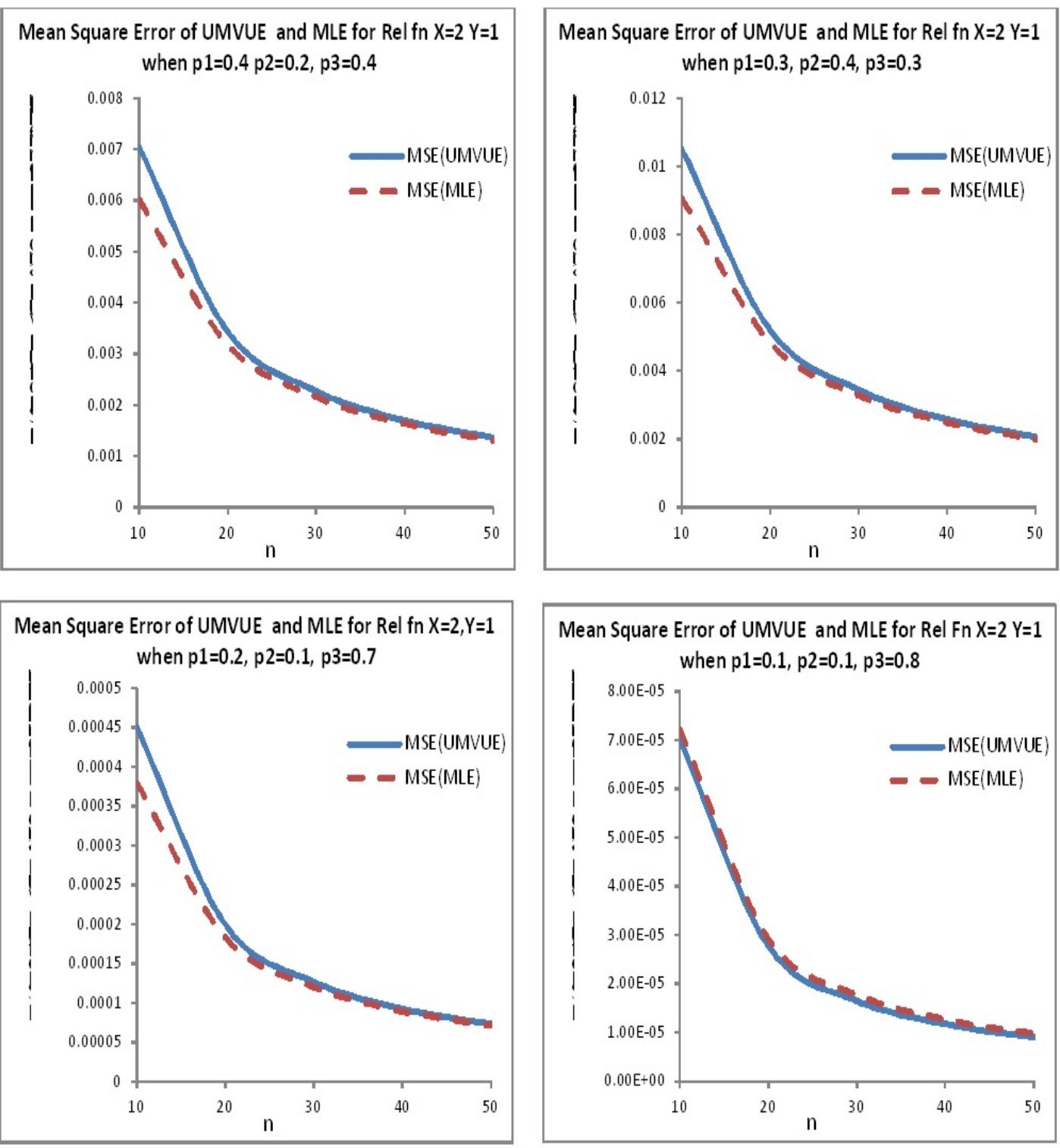

Fig. 10. 


\section{Example from Cricket's Indian Premium League(IPL2014)}

Consider the 2014 season of Cricket's Indian Premium League.(IPL 2014). A total of 154 players from around the world were chosen to participate as member of any one of the 8 playing teams. The first match played by every batsman was considered. A total of 98 batsmen became out during the course of the first match that they played. Data was collected from the ball by ball account of each of these 98 batsmen till they became out.

From this it was possible to obtain

(i) total number of balls faced by each player till he was out.

(ii) Number of balls hit that went beyond boundary ( as a 4 or a 6) prior to being out

(iii) Number of balls hit that did not go to boundary prior to being out.

Let

$X$ : The number of balls hit by a player that went beyond boundary( ie a 4 or 6 ) till he was out.

Y: The number of balls hit by a player that did not go beyond boundary till he was out.

Hence $p_{1}=\mathrm{P}\left(\right.$ Player hit a ball that went beyond boundary),$p_{2}=\mathrm{P}$ (Player hit a ball that did not go beyond boundary and he was not out $)$ and $p_{3}=\mathrm{P}(\mathrm{He}$ hit a ball and was out $)$.

Table 1. IPL 2014

\begin{tabular}{|c|c|c|c|c|c|c|c|c|c|c|c|c|c|c|c|c|c|c|c|c|c|}
\hline $\mathrm{X} / \mathrm{Y}$ & 0 & 1 & 2 & 3 & 4 & 5 & 6 & 7 & 8 & 9 & 10 & 11 & 12 & 13 & 14 & 15 & 16 & 17 & 18 & 19 & TOTAL \\
\hline 0 & 7 & 12 & 8 & 1 & 4 & 2 & 3 & 7 & 4 & 1 & 2 & 2 & 1 & 0 & 1 & 1 & 0 & 0 & 0 & 0 & 56 \\
\hline 1 & 2 & 3 & 3 & 0 & 1 & 1 & 2 & 1 & 2 & 2 & 1 & 2 & 0 & 0 & 0 & 0 & 0 & 0 & 0 & 2 & 22 \\
\hline 2 & 0 & 0 & 0 & 0 & 0 & 0 & 1 & 1 & 1 & 0 & 0 & 0 & 1 & 1 & 2 & 1 & 1 & 1 & 0 & 0 & 10 \\
\hline 3 & 0 & 0 & 0 & 0 & 0 & 0 & 0 & 0 & 0 & 0 & 1 & 0 & 0 & 0 & 0 & 0 & 0 & 0 & 1 & 0 & 2 \\
\hline 4 & 0 & 0 & 1 & 0 & 0 & 1 & 1 & 0 & 0 & 0 & 0 & 0 & 3 & 0 & 0 & 0 & 0 & 0 & 0 & 0 & 6 \\
\hline 5 & 0 & 0 & 0 & 0 & 0 & 0 & 0 & 0 & 0 & 0 & 0 & 0 & 0 & 0 & 0 & 0 & 0 & 0 & 0 & 0 & 0 \\
\hline 6 & 0 & 0 & 0 & 0 & 0 & 0 & 0 & 0 & 0 & 0 & 0 & 0 & 0 & 0 & 0 & 0 & 0 & 0 & 0 & 0 & 0 \\
\hline 7 & 0 & 0 & 0 & 0 & 0 & 0 & 0 & 0 & 0 & 0 & 0 & 1 & 0 & 0 & 0 & 0 & 0 & 0 & 0 & 0 & 1 \\
\hline 8 & 0 & 0 & 0 & 0 & 0 & 0 & 0 & 0 & 0 & 0 & 0 & 0 & 0 & 0 & 0 & 0 & 0 & 0 & 0 & 1 & 1 \\
\hline TOT & 9 & 15 & 12 & 1 & 5 & 4 & 7 & 9 & 7 & 3 & 4 & 5 & 5 & 1 & 3 & 2 & 1 & 1 & 1 & 3 & 98 \\
\hline
\end{tabular}

The following calculations have been made from the above table.

Table 2. Result

\begin{tabular}{|l|c|c|}
\hline & MVUE & MLE \\
\hline p1 & 0.10847 & 0.108343 \\
\hline p2 & 0.77057 & 0.769613 \\
\hline p3 & 0.120947 & 0.1220423 \\
\hline
\end{tabular}

Table 3. Result for Reliability Function when $\mathrm{X}=1, \mathrm{Y}=1$

\begin{tabular}{|c|c|c|}
\hline & MVUE & MLE \\
\hline 1 & 0.472826 & 0.47027027 \\
\hline 2 & 0.86433 & 0.86312849 \\
\hline 3 & 0.45810938 & 0.4554411031 \\
\hline
\end{tabular}


Table 4. Result for Reliability Function when $X=1, Y=2$

\begin{tabular}{|c|c|c|}
\hline & MVUE & MLE \\
\hline 1 & 0.472826 & 0.47027027 \\
\hline 2 & 0.7469119 & 0.7449907 \\
\hline 3 & 0.4340009 & 0.43122889 \\
\hline
\end{tabular}

Table 5. Result for Reliability Function when $X=2, Y=1$

\begin{tabular}{|c|c|c|}
\hline & MVUE & MLE \\
\hline 1 & 0.222202 & 0.22115413 \\
\hline 2 & 0.86433 & 0.86312849 \\
\hline 3 & 0.22062235 & 0.2195474801 \\
\hline
\end{tabular}

Table 6. Result for Reliability Function when $\mathrm{X}=2, \mathrm{Y}=2$

\begin{tabular}{|c|c|c|}
\hline & MVUE & MLE \\
\hline 1 & 0.222202 & 0.221154 \\
\hline 2 & 0.7469119 & 0.7449907 \\
\hline 3 & 0.21680711 & 0.215687648 \\
\hline
\end{tabular}

From the example of IPL2014, it is observed that both the UMVUE and MLE estimates calculated for $p_{1}, p_{2}, p_{3}$ and for reliability function are very close to each other. In Table2, $p_{1}=\mathrm{P}($ The batsmen hits a 4 or 6$)=$ Probability of a beyond boundary ball is 0.108 . $p_{2}=$ $\mathrm{P}($ Batsmen does not hit a 4 or 6 and is not out $)=$ Probability of a non-beyond boundary ball $=0.77$ which is larger than $p_{1}$. The probability $p_{3}=\mathrm{P}($ Batsmen hits and is out $)$ is very low and $=0.12$.

When we consider the reliability functions in Table 3, the probability of batsmen hitting atleast 1 beyond boundary ball is 0.47 whereas the probability of his hitting atleast 2 beyond boundary balls reduces to 0.222 as seen in Table 5.Similarly the probability of hitting atleast one non-beyond boundary ball is 0.86 from Table 3 whereas the probability of hitting atleast 2 balls that are non beyond boundaries reduces and is 0.75 from Table 4 .

Finally the probability of hitting both i.e. atleast one beyond boundary ball and atleast one nonbeyond boundary ball is 0.45 from Table2. This probability is larger than the probability of hitting atleast two balls that are beyond boundaries and atleast two that are non-beyond boundaries which is 0.22 from table 5. This reduction in probabilities as $\mathrm{X}$ and $\mathrm{Y}$ values increase corresponds to what actually happens in a game of cricket.

\section{Acknowledgements}

The authors are thankful to both the referees and Professor Hamedani for their valuable comments and suggestions. 


\section{References}

[1] Hare Krishna and Pundir,P.S.(2009).A Bivariate Geometric Distribution with Applications to Reliability. Commun.Statist Theor. Meth., 38:1079-1093.

[2] Phatak, A.G. and Sreehari, M(1981).Some characterizations of a Bivariate Geometric Distributions.Journal Indian Statistical Association 19:141-146.

[3] Downtown,F.(1970).Bivariate Exponential Distribution in Reliability Theory.JRSS B, 32:408-417.

[4] Kocherlakote,S.,Kocherlakote,K.(1992).Bivariate Discrete Distributions.New York: Marcel Dekker, Inc. 